Published in final edited form as:

Exp Eye Res. 2008 November ; 87(5): 400-401. doi:10.1016/j.exer.2007.12.008.

\title{
Focus on Molecules:
}

\author{
Human mucin MUC16
}

Beatriz H. Perez and llene K. Gipson ${ }^{*}$

Schepens Eye Research Institute and Department of Ophthalmology, Harvard Medical School, 20 Staniford Street, Boston, MA 02114, USA

\section{Keywords}

Mucins; MUC16; H185 antibody; siRNA; cancer

\section{Structure}

MUC16 is one of 10 membrane-associated mucins (MAMs) known to date, and it is the largest of the class. This heavily $O$-glycosylated glycoprotein is found on the apical membranes of the wet-surfaced epithelial cells of both the human cornea (Figure 1A) and conjunctiva; it is especially prominent specifically on the tips of surface microplicae (Figure 1B). It is also found on the surfaces of respiratory tract epithelia and female reproductive tract epithelia (for review see (Blalock, T. et al., 2007)). MUC16, also known as the CA125 antigen, was cloned from an ovarian cancer cell line, OVCAR-3, and was mapped to chromosome 19p13.2 (for review see (Gipson, I.K. et al., 2004)) [Accession number: Nucleotide: NM_024690, Protein:

Q8WXI7]. MUC16 has a lengthy core peptide of 22,152 amino acids, with a molecular weight of 2.5 MDa. As with other mucins of this class, MUC16 has a large ectodomain, estimated to extend up to $500 \mathrm{~nm}$ from the cell surface into the glycocalyx, a single-pass membranespanning domain, and a small cytoplasmic tail (Figure 1D). The ectodomain is composed of a heavily $O$-glycosylated $\mathrm{N}$-terminus, a tandem repeat region with over 60 tandem repeats of 156 amino acids each, and a region with several SEA domains near the membrane-spanning sequence (Figure 1) (for review see (Gipson, I.K. et al., 2004). The tandem repeats of amino acids common to all mucins (albeit each mucin gene's repeats are of different lengths) are rich in serine, threonine and proline-the serines and threonines being sites of $O$-glycan posttranslational additions. Due to the heavy $O$-glycosylation in the ectodomains of MAMs, up to $80 \%$ of the mass of the mucin is $O$-glycans, with galactose, $N$-acetylgalactosamine, $N$ acetylglucosamine, and sialic acids as the main sugars with minor amounts of fucose, mannose and glucose. The great number of $O$-glycans present in the ectodomain of membrane-associated mucins imparts to them a "bottle brush" tertiary conformation (for review see (Gipson, I.K. et al., 2004)).

The SEA domain region mediates an intracellular auto-cleavage and reassociation of the molecule in the endoplasmic reticulum, a process common to several MAMs, but whose

\footnotetext{
*Corresponding author, Ilene K. Gipson, Ph.D.: Mailing address (as above); Tel: 617-912-0210; Fax: 617-912-0126; E-mail: Ilene.Gipson@schepens.harvard.edu

First author, Beatriz Perez: Mailing address (as above); Tel: 617-912-0299; Fax: 617-912-0126; E-mail:

Beatriz.Perez@schepens.harvard.edu

Publisher's Disclaimer: This is a PDF file of an unedited manuscript that has been accepted for publication. As a service to our customers we are providing this early version of the manuscript. The manuscript will undergo copyediting, typesetting, and review of the resulting proof before it is published in its final citable form. Please note that during the production process errors may be discovered which could affect the content, and all legal disclaimers that apply to the journal pertain.
} 
significance is unknown. The ectodomain of MUC16 has been shown to be constitutively shed from the surface of epithelia, as it is detected in secretions of wet-surfaced epithelia, including the tears. To date, the mechanism and location of cleavage of the MUC16 ectodomain are unknown, but it appears that the intracellular cleavage and reassociation is not related to ectodomain shedding.

The cytoplasmic tail of MUC16 is not well characterized, but it contains several potential tyrosine phosphorylation sites, which are conserved in the mouse homolog of MUC16. The tail can associate by linking molecules with the actin cytoskeleton (Blalock, T. et al., 2007).

\section{Function}

Membrane-associated mucins are considered to be multifunctional molecules. On the one hand, they exhibit the classical mucin-associated functions of maintenance of hydration and lubrication of the epithelial surface, and the formation of a disadhesive barrier, all of which result from their heavy $O$-glycosylation. On the other hand, they can function as signaling molecules. For instance, MUC1, the most studied of the MAMs, has been shown to actively participate in signaling. It has been shown that a sequence in MUC1's cytoplasmic tail, SXXXXXSSL, interacts with $\beta$-catenin, and a fragment of the cytoplasmic tail is transported to the nucleus of the cell, thus, influencing the transcription of genes involved in cell proliferation and differentiation (for review see (Hollingsworth, M.A. and Swanson, B.J., 2004). Similarly, MUC16 is a multifunctional molecule. In studies using a human telomerase immortalized human corneal epithelial cell line in which MUC16 expression was stably knocked down using siRNA methods, adherence of Staphylococcus aureus to epithelial cells increases, indicating that the mucin prevents adhesion of pathogens. In addition, the mucin serves as a barrier to penetrance of agents into epithelial cells, since knockdown of the mucin increases penetrance of rose bengal dye (Blalock, T. et al., 2007). Rose bengal is used clinically to diagnose dry eye, as it penetrates areas of epithelium where protection has been compromised. These studies indicate that MUC16 is a key component of the ocular surface glycocalyx, where it plays a role in barrier formation on the surface of epithelial cells. Another function of MUC16 is indicated by studies of the cytoplasmic tail of MUC16, which demonstrate that a polybasic sequence of amino acids (RRRKK, conserved in the mouse homolog) within it, binds to the ezrin/radixin/moesin (ERM) family proteins. This association anchors the mucin to the actin cytoskeleton within microplicae on the surface of epithelia cells (Figure 1C). Although the function of the association of MUC16 with the actin cytoskeleton is unclear, it may facilitate surface cell membrane folding. Density of microplicae on cells of the ocular surface epithelium decreases as cells age at the surface, and they decrease in cells in dry eye (Blalock, T. et al., 2007).

\section{Disease involvement}

MUC16/CA125 has long been known to be an ovarian tumor cell marker, and more recently, it has been shown to be altered in dry eye. MUC16/CA125 levels in sera of women with ovarian cancer are elevated, and antibodies to the mucin, designated OC125, are widely used for detection of the cancer and for monitoring effects of treatment. Antibody therapies targeting MUC16 are under development for treatment of ovarian cancer (for review see(Hollingsworth, M.A. and Swanson, B.J., 2004).

In non-Sjögren's dry eye, alterations to MUC16 on the ocular surface have been demonstrated using the monoclonal antibody $\mathrm{H} 185$ that recognizes an O-acetylated carbohydrate epitope on the mucin. The decrease in binding of the antibody to the ocular surface increases with severity of the disease (Gipson, I.K., 2007). It is not known if the alteration in MUC16 seen in dry eye is a result of decrease in expression of the mucin, an increase in shedding of its ectodomain from the epithelial surface, or a change in glycosylation of the mucin, resulting in the 
diminishment of the H185 epitope. In any event, alteration of the mucin may be directly correlated to the increase in rose bengal dye penetrance seen in the drying diseases, as well as the increase in infections seen in the disease.

\section{Future studies}

Although much has been learned in recent years about the membrane-spanning mucin MUC16, there are major gaps in knowledge regarding the basic function of the molecule and its role in ocular surface disease.

Future studies to determine the signaling capabilities of its cytoplasmic tail as well as the mechanism of the shedding of its extracellular domain will yield basic information relevant, not only to dry eye, but also potentially to methods of pathogen invasion.

Clinical studies sorting out the nature of the alterations of the mucin in dry eye may yield therapeutic opportunities for drying eye disease. Perhaps inflammatory mediators that are present in the tears of dry eye patients mediate shedding of the mucin, which results in areas of dye penetrance seen in the disease. On the other hand, a change in glycosyltransferase activity in the disease may alter MUC16 glycosylation, and thus, the physical character of the mucin's extracellular domain. Focus on MUC16 in ocular surface biology and disease is a promising avenue for future research.

\section{References}

Blalock T, Spurr-Michaud S, Tisdale A, Heimer S, Gilmore M, Ramesh V, Gipson I. Functions of MUC16 in corneal epithelial cells. Invest Ophthalmol Vis Sci 2007;48:4509-4518. [PubMed: 17898272]

Gipson IK. The ocular surface: the challenge to enable and protect vision: the Friedenwald lecture. Invest Ophthalmol Vis Sci 2007;48:4390-4398. [PubMed: 17898256]

Gipson IK, Hori Y, Argueso P. Character of ocular surface mucins and their alteration in dry eye disease. Ocul Surf 2004;2:131-148. [PubMed: 17216084]

Hollingsworth MA, Swanson BJ. Mucins in cancer: Protection and control of the cell surface. Nat Rev Cancer 2004;4:45-60. [PubMed: 14681689] 

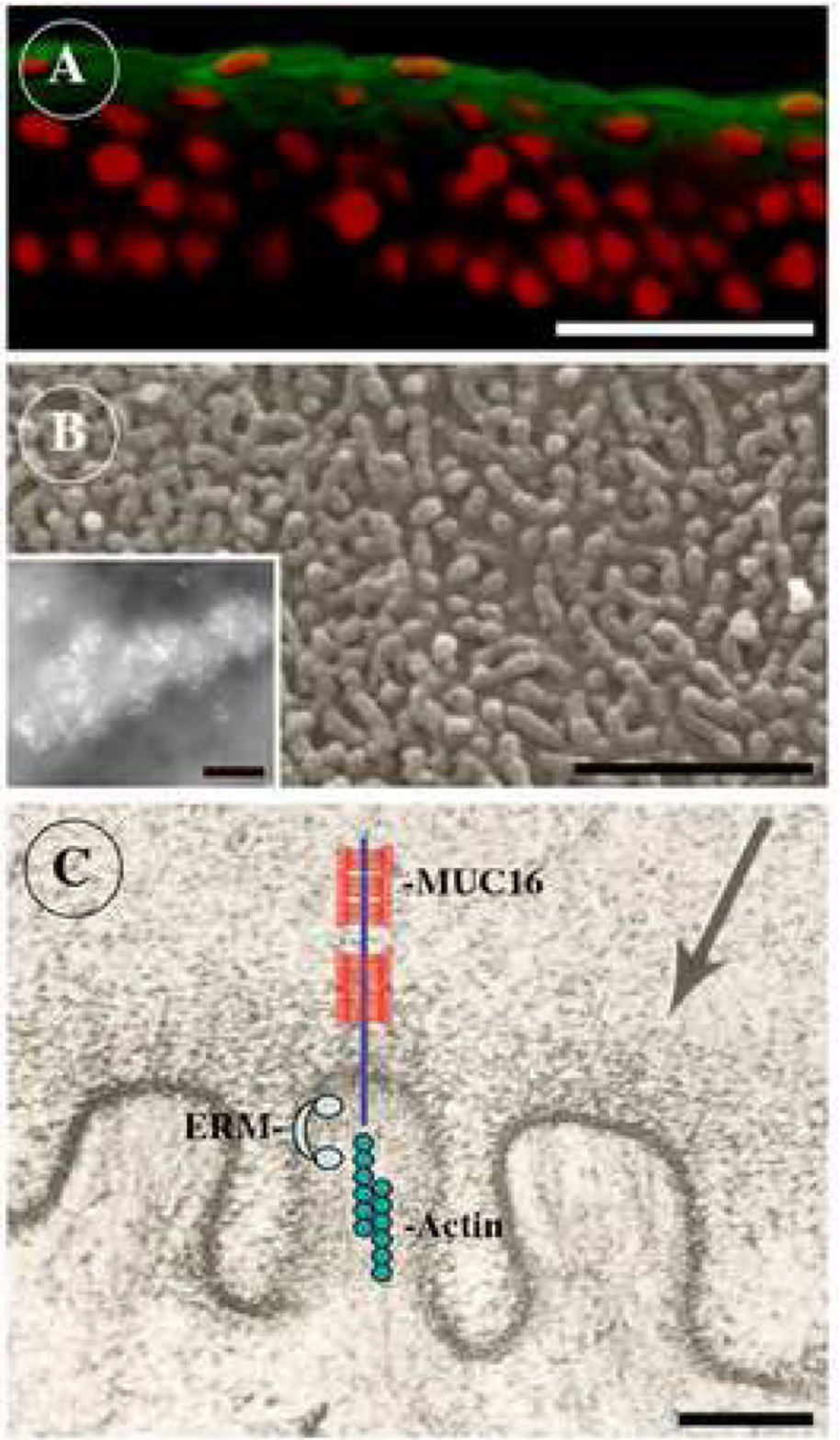

Fig. 1.

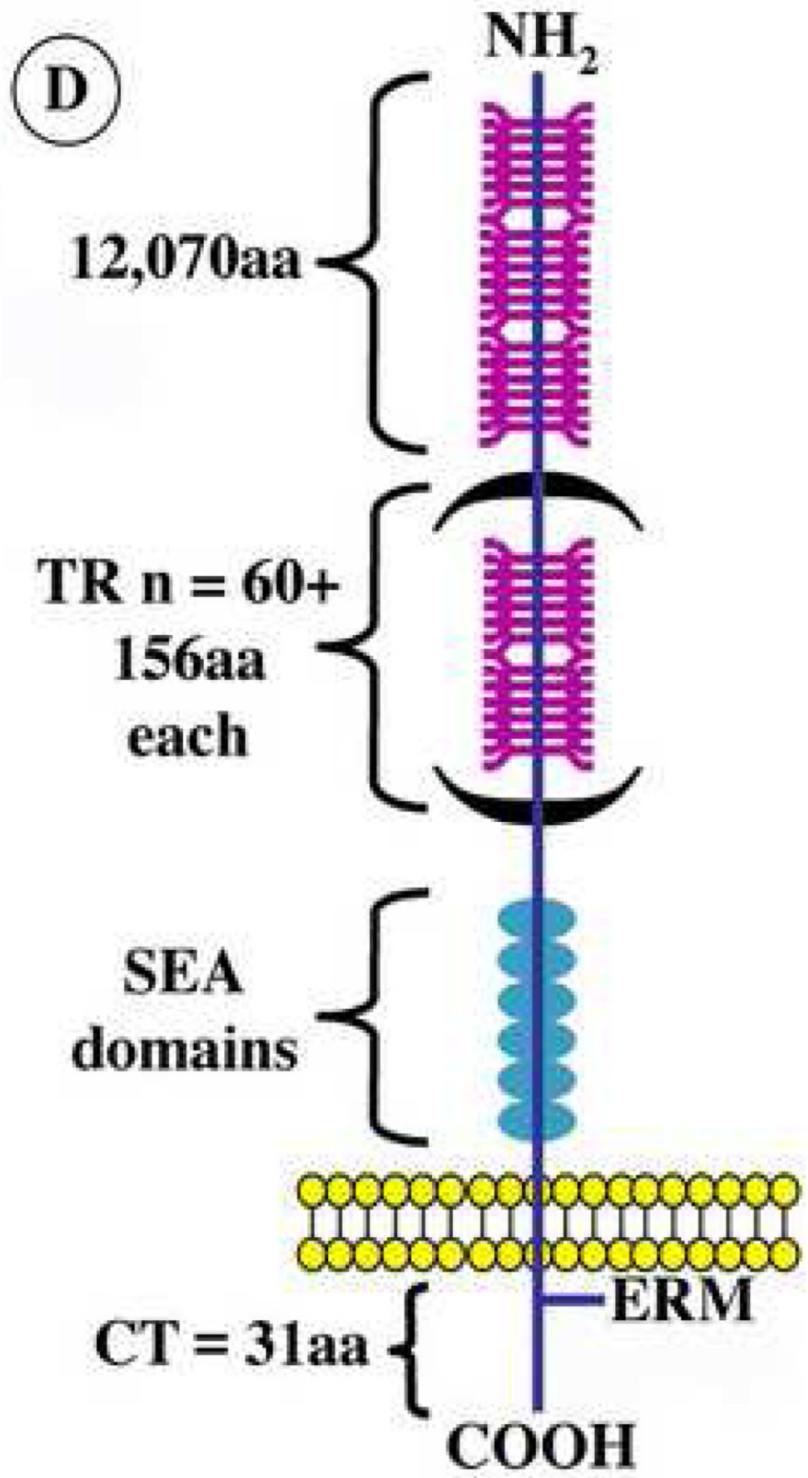

Micrographs and diagrams demonstrating the localization and structure of MUC16. (A) Light micrograph of corneal epithelium showing localization of MUC16 protein (M11 antibody) on apical cells (green), nuclei are counterstained with propidium iodide (red). (B) Scanning electron micrograph showing localization of MUC16 on the microplicae on the cornea and an inset field emission scanning electron micrograph showing immunogold localization of MUC16 to the microplicae. (C) Transmission electron micrograph of microplicae showing the electron dense zone of the glycocalyx on the tips of the microplicae (arrow). Superimposed on the micrograph is a diagram of MUC16 showing its glycosylated ectodomain (red) and its cytoplasmic association to the actin cytoskeleton which is brought about by linkage to ERM's (ezrin, rodoxin, moesin, and merlin) a family of proteins involved in formation of surface membrane specializations. (D) Diagram showing structural motifs of MUC16. The ectodomain is composed of a heavily glycosylated region at the $\mathrm{NH}_{2}$ terminus followed by over 60 tandem repeats (TR) of amino acids rich in serine and threonine and by a SEA domain region (blue). 
MUC16 crosses the cell membrane (yellow) and its short cytoplasmic tail has several potential tyrosine phosphorylation sites as well as an ERM binding domain. Scale bar: (A) $50 \mu \mathrm{m}$; (B) $1 \mu \mathrm{m}$ (insert) $0.1 \mu \mathrm{m}$; (C) $0.1 \mu \mathrm{m}$. 